\title{
Teilnehmerrekord! neuroRAD 2019 rückt Zukunftsthemen in den Blick
}

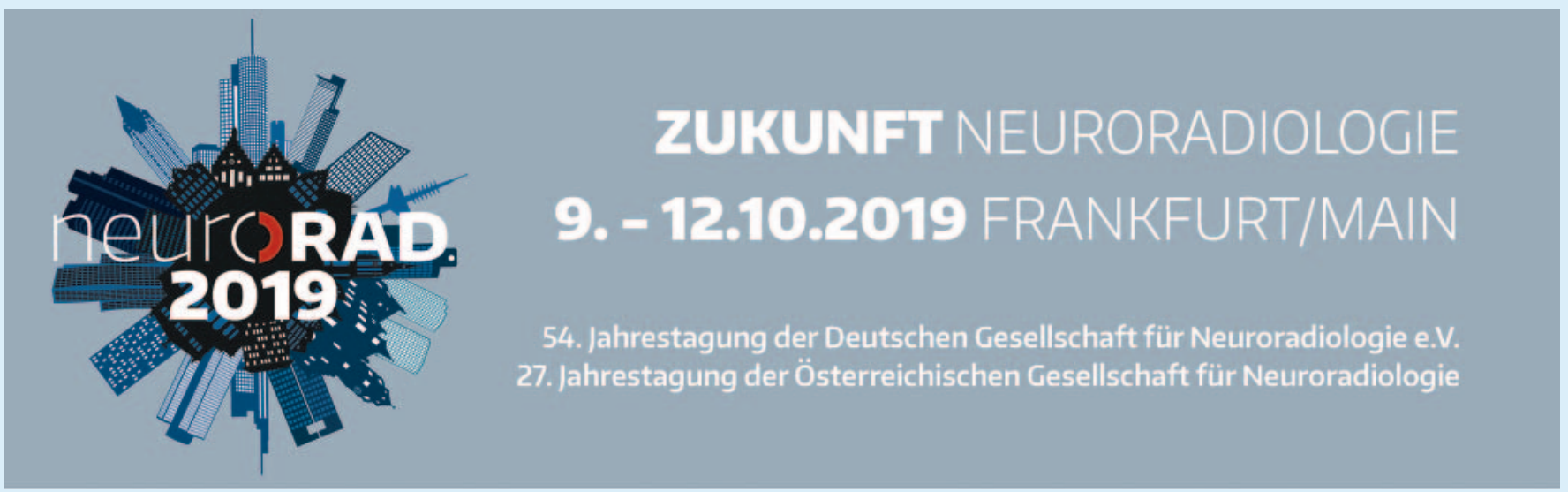

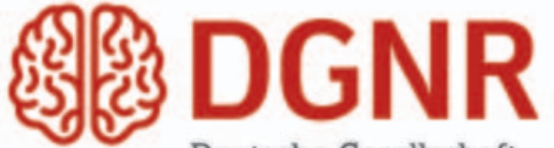

Deutsche Gesellschaft

für Neuroradiologie e.V.

Die 54. Jahrestagung der Deutschen Gesellschaft für Neuroradiologie und 27. Jahrestagung der Österreichischen Gesellschaft für Neuroradiologie war ein voller Erfolg. Beinahe 1100 Teilnehmerinnen und Teilnehmer aus 26 Nationen haben ihren Weg nach Frankfurt gefunden und vier spannende Kongresstage erlebt. Motto des diesjährigen neuroRAD war „Zukunft Neuroradiologie“. Die Themen Künstliche Intelligenz, Machine Learning \& Co. zogen sich durch das gesamte Kongressprogramm und alle Teilnehmenden in den Bann.

\section{Neuer Rekord: 1100 Teilneh- merinnen aus 26 Ländern}

Der neuroRAD 2019 hat einen neuen Teilnehmerrekord aufgestellt: 1094 Besucherinnen und Besucher aus 26 verschiedenen $\mathrm{Na}$ tionen waren in Frankfurt vor Ort. Auch die Workshops, die neue StAR-Lounge und das erstmals stattfindende Format der PowerPitches waren durchweg sehr gut besucht.

\section{Congress on demand}

Über DGNR.org > Fachinfos > congress on demand können Sie noch einmal die einzelnen Beiträge Revue passieren lassen.

Und gerade dann, wenn Sie primär nicht die Möglichkeit hatten, den entsprechenden Vortrag in Frankfurt zu hören. Es gab wirklich viele hervorragende Präsentationen, dass Sie sich diese Möglichkeit keinesfalls entgehen lassen sollten!

\section{Preisträger und neue}

\section{Ehrenmitglieder 2019}

Traditionell wurden auch wieder zahlreiche Preise vergeben. Wir gratulieren allen Preisträgern sehr herzlich. Neues Ehrenmitglied der DGNR ist Herr Prof. Dr. Gerhard Schroth (Universitätsspital Bern). Neues Ehrenmitglied der ÖGNR ist Herr Prof. Dr. Georges Rodesch (Hôpital Foch, Paris). Im Rahmen des neuroRAD 2019 wurde auch der Arthur Schüller Preis der ÖGNR verliehen.

Eine Übersicht aller Preisträger finden Sie auf DGNR.org > News > Preisträger und Ehrenmitglieder 2019

\section{Fotogalerien neuroRAD 2019}

Der neuroRAD 2019 bot zahlreiche Höhepunkte, die einer Erinnerung wert sind. An allen vier Tagen begleitete ein professioneller
Fotograf die Geschehnisse im Kap Europa in Frankfurt und hat neben vollen Sälen, großen Namen, spektakulärer Architektur, stolzen Preisträgern auch den ein oder anderen Schnappschuss festgehalten. Schauen Sie rein.

Zahlreiche Fotogalerien finden Sie unter DGNR.org > News

\section{Impressionen vom \\ neuroRAD 2019}

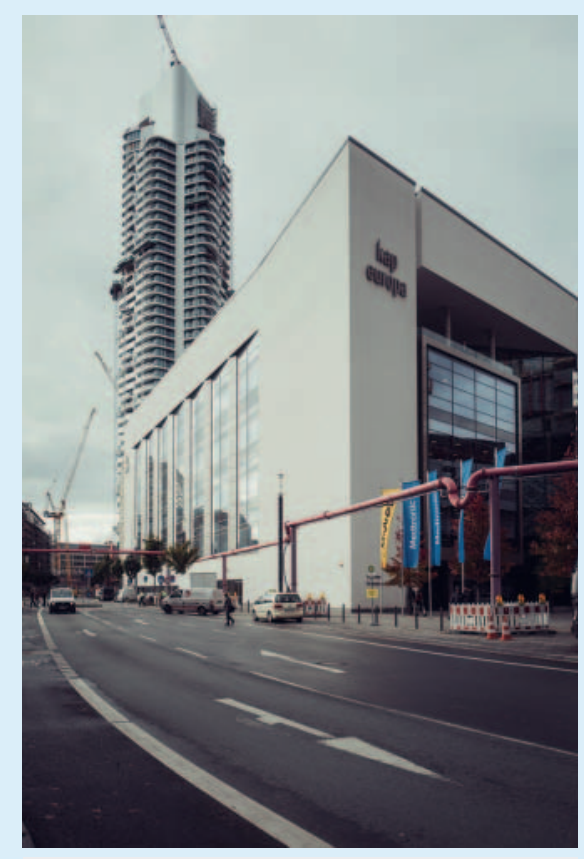

Die Heimat des neuroRAD seit 2018: das Kap Europa in Frankfurt am Main. (c) offenblen.de/DGNR. 


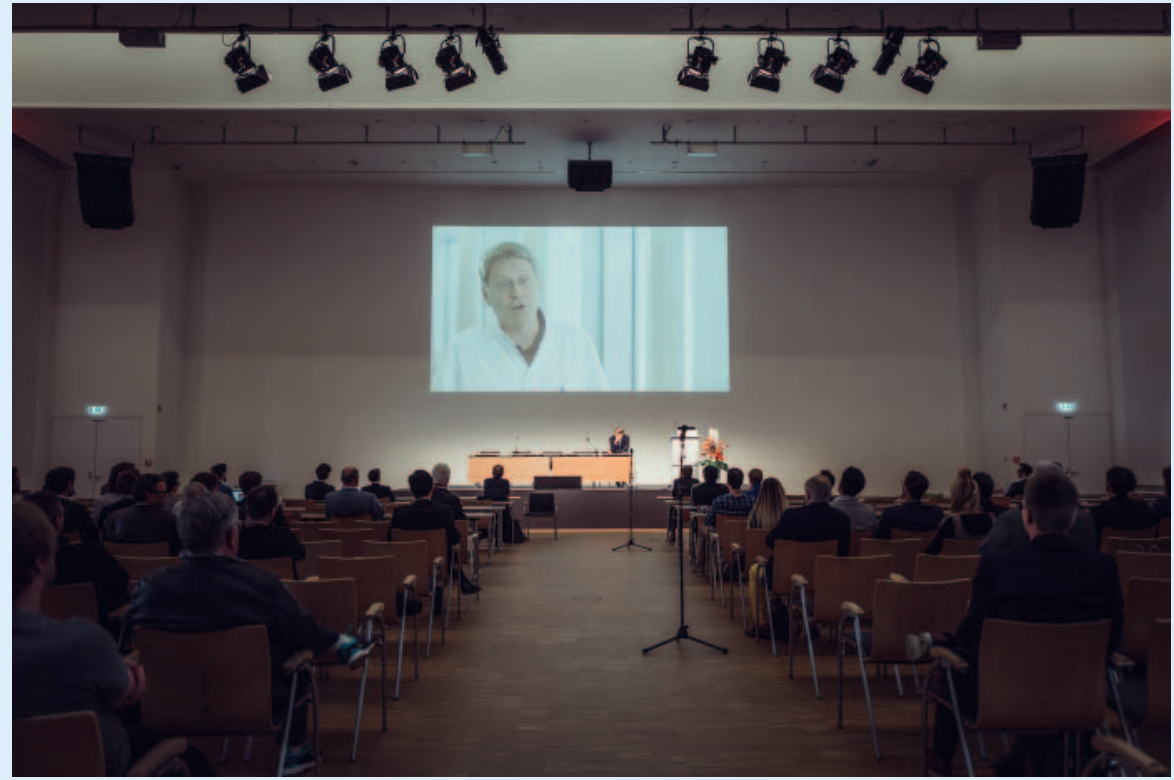

Der neue Imagefilm „Neuroradiologie“ der DGNR feierte in Frankfurt Premiere. (c) offenblen.de/DGNR.

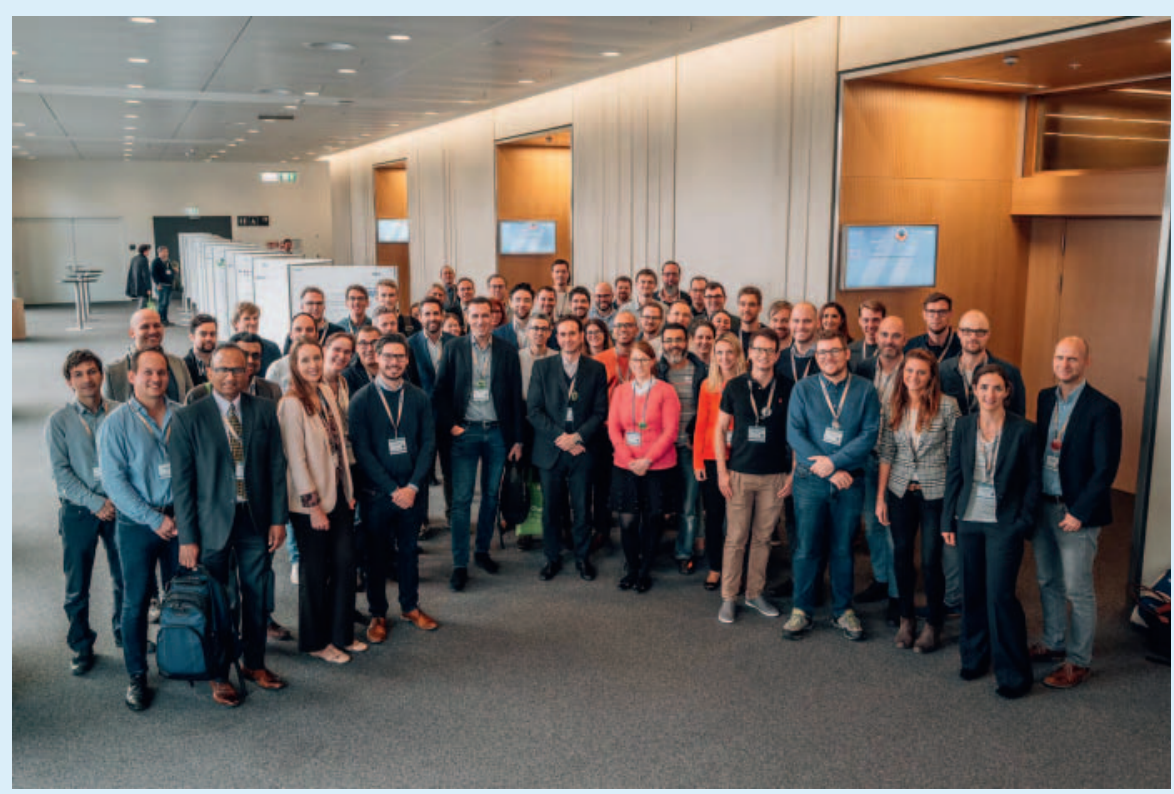

Die zweite Ausgabe der German Stroke School auf dem neuroRAD 2019 war ein voller Erfolg. Über 50 Teilnehmerinnen und Teilnehmer aus dem In- und Ausland haben an zwei Tagen in der Welt der Behandlung des ischämischen Schlaganfalls wichtige Einblicke und Fertigkeiten erfahren. Fortsetzung in 2020 garantiert! @ offenblen.de/DGNR.

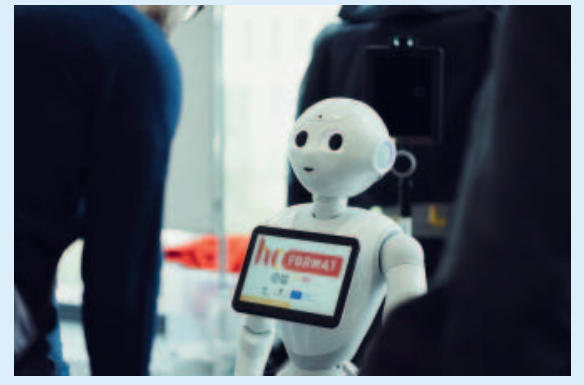

Ein ganz besonderer Gast im MTRA-Programm war Pepper, der Pflegeroboter der Uni Halle. @ offenblen.de/DGNR.

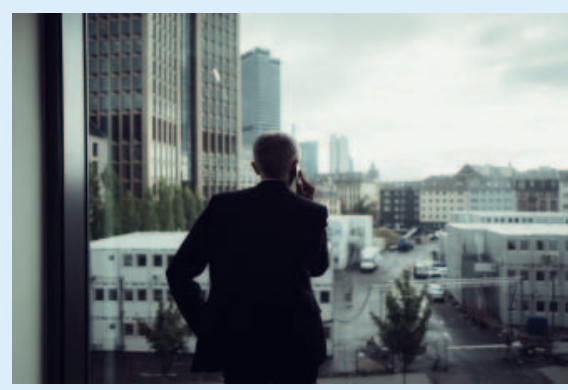

Der neuroRAD - DER alljährliche Treffpunkt von Neuroradiologinnen und Neuroradiologen aus Deutschland und der ganzen Welt. @ offenblen.de/DGNR.

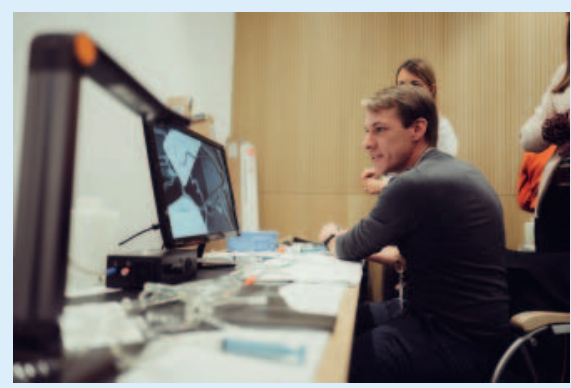

Workshops und Hands-on-Kurse werden beim neuroRAD schon immer groß geschrieben. $\odot$ offenblen.de/DGNR.

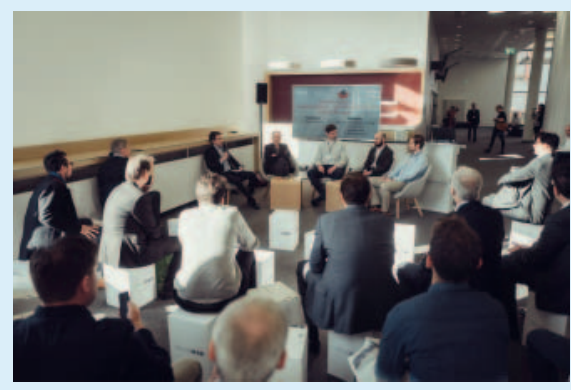

Die StAR-Lounge („Start-Up and Applied Research“) feierte Premiere auf dem neuroRAD 2019. Zahlreiche Start-ups und junge Firmen präsentierten hier Ihre Innovationen zu KI \& Co. @ offenblen.de/DGNR. 


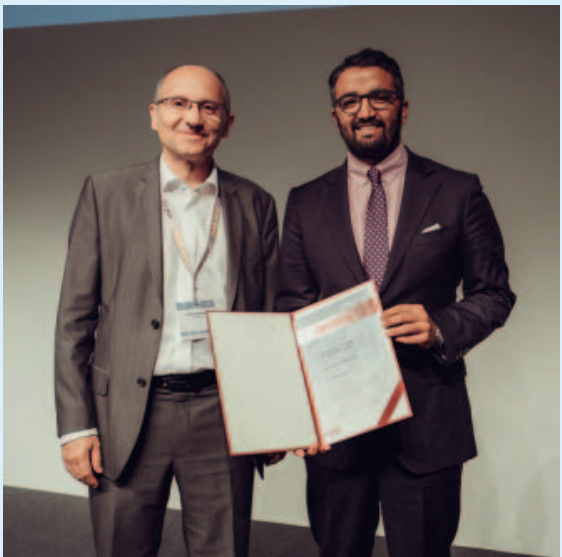

Dr. Saif Afat (re) mit Prof. Dr. Martin Wiesmann bei der Preisverleihung auf dem neuroRAD 2019. @ offenblen.de/DGNR.

\section{Preisträger 2019}

\section{Ehrenmitglied 2019}

Prof. Dr. Gerhard Schroth, Universitätsspital Bern

\section{Marc-Dünzl-Preis 2019}

Dr. Saif Afat, Universitätsklinikum Tübingen für seine Arbeit „Diagnostic Accuracy of Simulated Low-Dose Perfusion CT to Detect Cerebral Perfusion Impairment after Aneurysmal Subarachnoid Hemorrhage: A Retrospective Analysis“

\section{Kurt-Decker-Preis 2019}

PD Dr. Gabriel Broocks, Universitätsklinikum Hamburg-Eppendorf

für seine Arbeit „Quantitative Lesion Water Uptake - Validation and Clinical Application of a Novel Computed Tomography-Based Imaging Biomarker in Ischemic Stroke“

PD Dr. Dipl.-Inf. Julian Caspers, Universitätsklinikum Düsseldorf

für seine Arbeit „Methodik und Anwendung bildgebungsgestützter Verfahren zur Untersuchung funktioneller Hirnnetzwerke“

PD Dr. Benedikt Wiestler, TU München für seine Arbeit „Neuroradiologie 4.0“

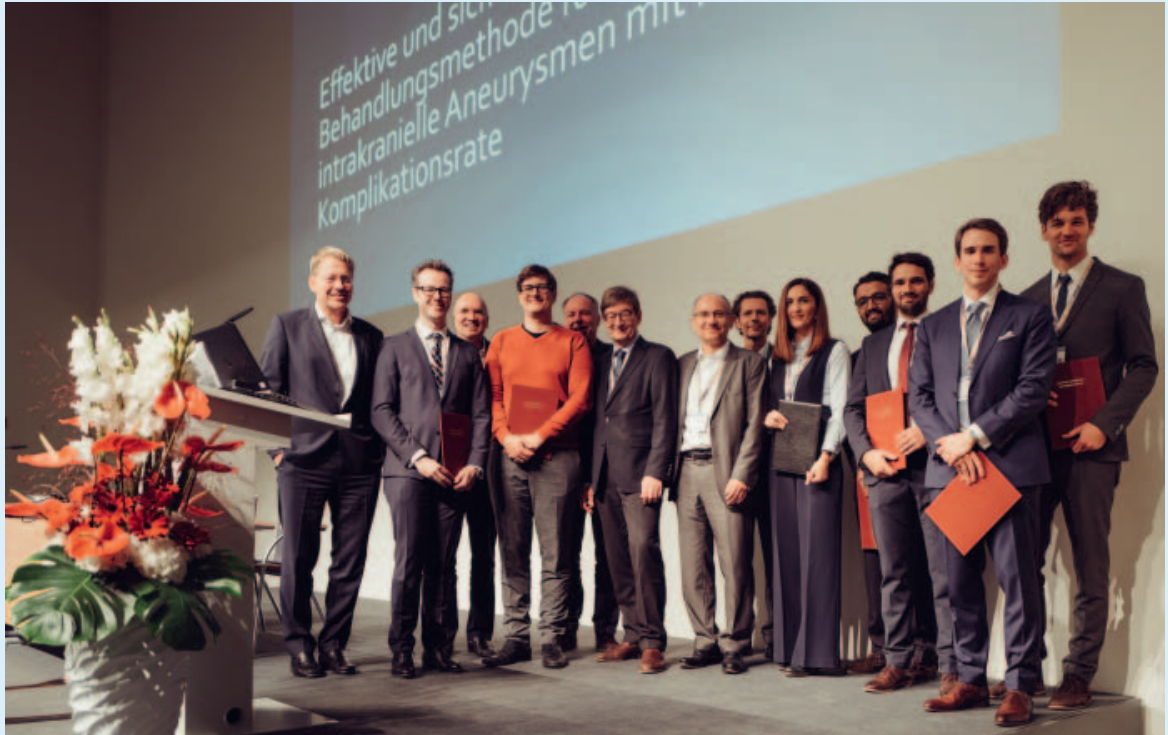

Gruppenfoto aller Preisträger und Laudatoren auf dem neuroRAD 2019 in Frankfurt am Main. (c) offenblen.de/DGNR.

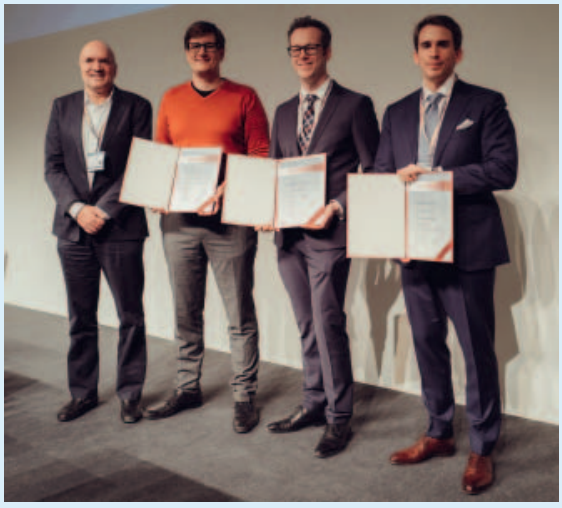

(v. re n. li) PD Dr. Gabriel Broocks, PP Dr. Dipl.-Inf. Julian Caspers, PD Dr. Benedikt Wiestler, Prof. Dr. Horst Urbach (DGNRPräsident). (C) offenblen.de/DGNR.

\section{DGNR-Interventionspreis 2019}

PD Dr. Peter Sporns, Universitätsklinikum Münster

für seine Arbeit „Feasibility, Safety and Outcome of Endovascular Recanalization in Childhood Stroke (Save ChildS): a multicenter retrospective observational study“

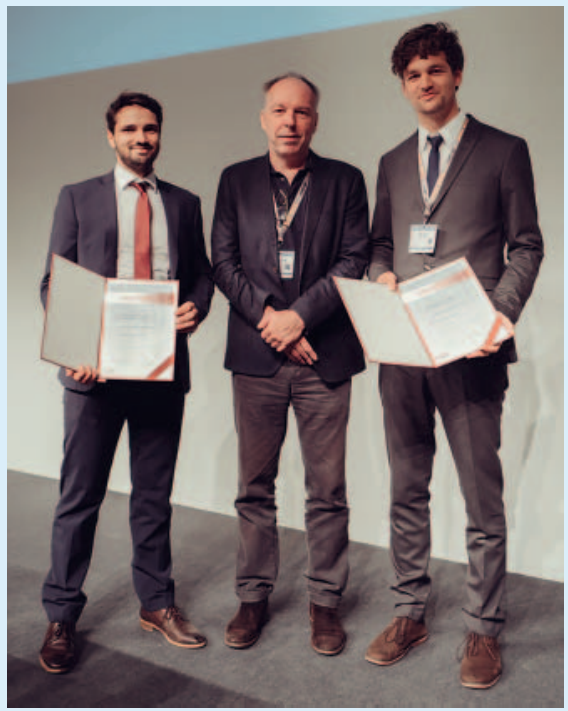

(v. li n. re): Dr. Dominik Vollherbst, Prof. Dr. Michael Forsting, PD Dr. Peter Sporns. (c) offenblen.de/DGNR.

Dr. Dominik Vollherbst, Universitätsklinikum Heidelberg

für seine Arbeit „Neue Behandlungstechniken für die endovaskuläre Embolisation intrakranieller Gefäßmalformationen“ 


\section{Posterpreisträger}

\begin{tabular}{|c|c|c|}
\hline Preisträger & Ort & Abstract \\
\hline Frau Dr. Silke Hopf-Jensen & Flensburg & Neurophysiological Monitoring in the Endovascular Therapy of Aneurysms \\
\hline Herr Dr. Nico Sollmann & München & $\begin{array}{l}\text { Tube current reduction in computed tomography angiography - how low can we go in patients with } \\
\text { suspected acute stroke? }\end{array}$ \\
\hline Frau Desiree Grosser & Hannover & Korrelation von radiologischen Parametern bei Morbus Menière \\
\hline Herr Amr Farahat & Magdeburg & Implicit motor learning modulates functional connectivity of DBS therapeutic target for tremor. \\
\hline Herr Samuel Voss & Magdeburg & $\begin{array}{l}\text { Combining high-resolution vessel wall imaging with image-based flow evaluation in unruptured middle } \\
\text { cerebral aneurysms }\end{array}$ \\
\hline Herr Prof. Dr. Bernd Turowski & Düsseldorf & Mikrokatheter-Rotation bei Rückzug in der distalen ACI \\
\hline Frau Mariya Pravdivtseva & Kiel & Do my vessel implants work? An answer attempted with MRI \\
\hline Herr Andreas Georg Junge & Heidelberg & $\begin{array}{l}\text { How to break the bottleneck of Al-driven radiology and get your hands on the new gold: "RIPE" a solution } \\
\text { for data extraction and analysis pipeline }\end{array}$ \\
\hline Frau Dr. Jessica Jesser & Heidelberg & $\begin{array}{l}\text { Stroke lesion topography and its relation to severity of NIHSS and early recovery potential in acute left } \\
\text { MCA ischemia }\end{array}$ \\
\hline
\end{tabular}

\section{Preis des Vereins für Hirn-Aneurysma-Erkrankte}

Johannes Hensler

für seinen Vortrag: „MR vessel wall imaging intrakranieller Aneurysmen nach endovaskulärer Therapie“

Theo Demerath für sein Poster: „High resolution vessel wall imaging involving $3 \mathrm{~T}$ compressed sensing (CS) time-of-flight MRA in a case of acute intracranial anterior circulation“

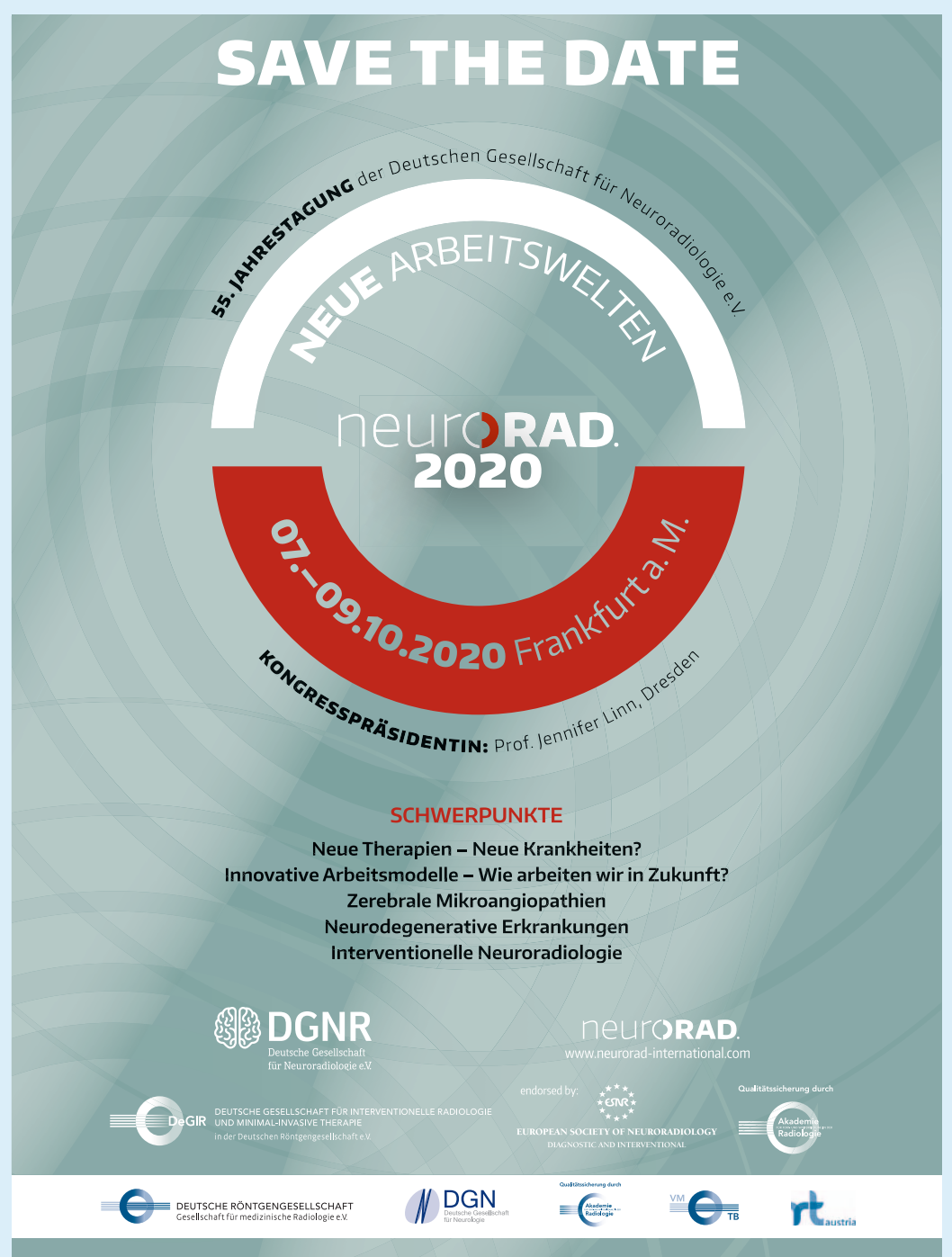

